\title{
Perceptual orientational asymmetries: A comparison of visual and haptic space
}

\author{
EUGENE C. LECHELT, JAMES ELIUK, and GORDON TANNE \\ University of Alberta, Edmonton, Alberta, Canada T6G $2 E 9$
}

\begin{abstract}
Stimulus orientation discrimination was investigated in visual and haptic modalities under conditions of simultaneous matching and memory. Discrimination of vertical and horizontal was significantly more accurate than discrimination of oblique stimulus orientations $\left(45^{\circ}, 135^{\circ}, 225^{\circ}\right.$, and $315^{\circ}$ ) for both modalities; haptic errors, however, were significantly greater at each orientation. While subjects were reliably more accurate in visually matching oblique stimulus orientations to a standard than producing them from memory, for the haptic modality, differences between memory and matching conditions were less consistent across the orientations sampled.
\end{abstract}

Visual spatial discrimination is known to be a function of stimulus orientation. Jastrow (1893) was among the first to report visual orientational asymmetries in perceptual preferences and discriminations; he found that performance on a variety of perceptual tasks was clearly superior when stimulus arrangements were in horizontal or vertical orientations. This pervasive visual finding has been called the "oblique effect" and has been demonstrated in animal behavioral studies and in human children and adults (Apelle, 1972).

Although explanations of the visual "oblique effect" have emphasized either the role of the individual's ecological environment, i.e., a culturallearning explanation (Ross, 1974), or endogenous, neurological factors (Campbell \& Maffei, 1970; Mansfield, 1974), Annis and Frost's (1973) finding of different orientational anisotropies in visual acuity for groups reared in different ecological environments, however, suggests that the most parsimonious explanation for the visual "oblique effect" is an eclectic one assuming that orientation-specific neuronal detectors are tuned by early visual experience. It would seem that the demonstration of perceptual orientational asymmetries in other modalities in individuals raised in similar ecological environments could be important in assessing the credibility of a cultural-learning, neurological, or mutual bases for spatial orientational anisotropic effects.

While the supremacy of visual discrimination in vertical and horizontal orientations has been reported for several responses (see Appelle, 1972), documentation of the "oblique effect" in other modalities has yet to be clearly demonstrated. Other than in vision, the existence of spatial anisotropies

This research was supported by a grant (A8621) from the National Research Council of Canada to E. C. Lechelt. would most likely be exhibited in the haptic modality (Geldard, 1970). Also important is the fact that identical stimulus arrangements and procedures can be employed in studying visual and haptic spatial discrimination, thus permitting direct intermodal data comparisons. The present experiment will test for comparable perceptual orientational asymmetries in visual and haptic modalities using equivalent experimental arrangements and subjects of comparable age and raised in approximately equivalent ecological environments. Such a comparison, in addition to providing data to test communalities between the "acquisition" or "endogenous" bases for the "oblique effect" in visual and haptic modalities, would also seem to be significant both theoretically, i.e., is comparable stimulus information invariant across the two sensory systems as Gibson (1966) would suggest, and empirically, i.e., if the physical stimulus conditions are the same for both modalities, are they disposed to perceive and process the stimulus information in equivalent manners and with equal veridicality? The later concern also embraces the issue of the relationship between intermodal stimulus equivalence and perceptual equivalence and whether or not stimulus input, regardless of modality, is referred to a common perceptual mechanism (Brumaghim \& Brown, 1968).

A concomitant interest of the present experiment is a comparison of stimulus orientation discrimination under memoric and perceptual response conditions. Consistently, researchers have reported that the "oblique effect" is more pronounced when two stimuli, to be compared in terms of their orientation, are presented successively rather than simultaneously. Rudel and Teuber (1963) found that although children could readily discriminate vertical from horizontal lines, they confused oblique lines oriented in opposite directions, and errors increased reliably when the two lines were presented successively rather than simultaneously. Similarly, Bryant (1969) 
reported that children were able to distinguish among vertical, horizontal, and oblique lines when any two were presented simultaneously, but easily confused oblique line orientations when presented successively; vertical and horizontal orientations, however, were equally reliably discriminated for both simultaneous and successive conditions. Bryant (1969), in agreement with the suggestion of Over and Over (1967) that the "oblique effect" may be due largely to memory variables, concluded that the direction of slope of oblique lines is not encoded in memory.

Differences between simultaneous stimulus matching or discrimination and successive stimulus comparison are not surprising as they represent two different modes of response. Bartley (1969) considers that the difference between immediate matching of two coexistent stimuli or a comparison of them after being presented successively reflects perceiving and judging, respectively, with judgment responses always being more readily altered by memoric influence. Bartley (1972) further suggests that visual and haptic modalities differ basically in stimulus information reception, visual exploration of a stimulus complex being relatively more simultaneous than the successive sampling of stimulus detail in active haptic exploration.

Perceptual and memoric response conditions will be employed in the present experiment by having subjects match the orientation of two temporally coexistent stimuli (simultaneous matching) or produce specific stimulus orientations from memory, respectively. This manipulation will provide for an additional modality comparison, i.e., if spatial orientational asymmetries do exist in both visual and haptic modalities, are the magnitudes of these asymmetries constant across perceptual (simultaneous) and memoric (successive) response conditions? Also, as previous stimulus orientation research has almost exclusively tested orientations within the range of $90^{\circ}$ on either side of the upright vertical plane (upper right and left quadrants), a more extensive range of stimulus orientations will be sampled in this study to examine the pattern of orientational asymmetries throughout the upright frontal-plane quadrants.

\section{METHOD}

\section{Subjects}

Fourteen males and 14 females served as observers in this experiment as part of a course requirement.

\section{Visual Stimuli}

The visual stimuli were two $1 / 4 \times 12 \mathrm{in}$. luminous cardboard blades mounted on a $32 \times 55$ in. plywood frame painted in flat black. Each blade was attached at its center to an axle protruding through the frame and connected to a Selsyn motor behind the frame. The protruding axles were centered vertically on the frame and separated approximately 30 in. horizontally. To insure equivalent blade luminescence throughout the experi- ment, a Spectroline ultraviolet light source was appropriately positioned below and in front of the frame so as to provide equal luminance to each blade surface. Behind the plywood frame, $360^{\circ}$ protractors were attached to the same axles of the Selsyn motors on which the blades were mounted: this enabled the experimenter to determine the exact position of either blade.

The subjects sat at a table $12 \mathrm{ft}$ from the blades. Their heads were fixed in a chin- and foreheadrest which was adjusted slightly for each subject so that the blades were at constant eye level. Each blade subtended approximately $5^{\circ}$ of visual angle. Positioned on the right and left of the table were two Selsyn motors with protruding axles and attached knobs which the subjects used to manipulate the position of either stimulus blade. Through appropriate yoking of the Selsyn motors, the blades could be rotated independently in either direction by the experimenter or subject.

\section{Haptic Stimuli}

To provide for equivalent stimulus conditions, two $1 / 4$-in.-diam $\times 12$-in. aluminum rods were mounted on a similar frame in an identical spatial relation to the luminescent blades in the visual condition. Axles connected the rods in front of the frame to $360^{\circ}$ protractors behind the frame. The subjects or the experimenter could manually turn the rod or protractor, respectively, in order to manipulate rod position. A clamping device behind the frame permitted the experimenter to fix and hold either rod in any desired orientation.

The subjects sat directly between and approximately 18 in. from the aluminum rods. The entire frame was raised or lowered so the axes of the rods were alway at shoulder height.

\section{Procedure}

Visual condition. The subjects were first shown the operation of the apparatus and told they would be requested to produce or match certain orientations of the blades by appropriately turning the knobs on the motors situated on the table where they would sit during the experiment. The luminescent blades were positioned at each of the six orientations used in the experiment $\left(0^{\circ}, 45^{\circ}, 90^{\circ}, 135^{\circ}, 225^{\circ}, 315^{\circ}\right)$, and the subjects were informed that these orientations represented the bisections of each quadrant as well as vertical and horizontal planes. The subjects were then asked to position their heads in the rest and to maintain that position throughout the experiment. Room lights were then turned off and the experiment began approximately 2 min later. The experimental room was completely dark and only the luminescent blades were visible to the subjects.

Each of the six orientations were presented in random order three times, i.e., 18 random order presentations of orientation, for both memory and simultaneous matching conditions. The method of adjustment was used throughout the experiment. In the memory condition, one blade was covered and the experimentor asked the subjects to position the visible blade at one of the orientations, each orientation being randomly tested three times. In the matching condition, the experimenter set one of the blades at one of the six orientations and the subjects adjusted the other blade to be at an equivalent orientation. The right/left position of the standard blade orientation in the matching condition was balanced so that again each standard orientation was randomly presented three times first to the right and then to the left side. Thus, each subject made a total of 54 adjustments, i.e., 18 memory adjustments and 18 simultaneous matchings with the standard on the right and 18 matchings with the standard on the left.

Haptic condition. The subjects were again shown the apparatus and told that the task was exactly the same as in the visual condition only that they would be blindfolded and asked to manually turn the appropriate rod to the appropriate orientation. Haptic data collection was identical to visual, with each subject again making 54 orientation adjustments. In the memory condition, the subjects used the left hand to position one rod at an orienta- 
tion specified by the experimenter; each of the six orientations was randomly tested in this manner three times, i.e., there were 18 adjustments in random order. In the simultaneous matching condition, the six orientations were also randomly tested three times with the standard, fixed orientation occurring an equal number of times first on the right and then on the left side. When the standard rod was on the left, the subjects felt it with the left hand and adjusted the right rod with the right hand to be at the same orientation; when the standard was on the right, the subjects adjusted the left rod with the left hand to match the standard orientation of the right rod felt with the right hand.

While starting modality was randomized such that one-half of the subjects (seven males and seven females) began with the visual condition and half with the haptic condition, within each modality memory adjustments were made before simultaneous matchings. At no time was any feedback provided. The subjects were permitted as much time as required to make as accurate a response as possible. Most experimental sessions lasted approximately $40 \mathrm{~min}$, with a short break when alternating between modalities.

\section{RESULTS}

Data collected were the produced (memory and matching) orientations, to the nearest degree. Both the mean errors of produced orientations taking sign of direction of error into account, i.e., over- and underestimation, and the mean absolute error of produced orientations were calculated for each experimental condition and are presented in Figures 1 and 2, respectively. Figure 1 shows that orientations were both overestimated and underestimated with no consistent or systematic pattern, except that horizontal and vertical orientations were generally pro-

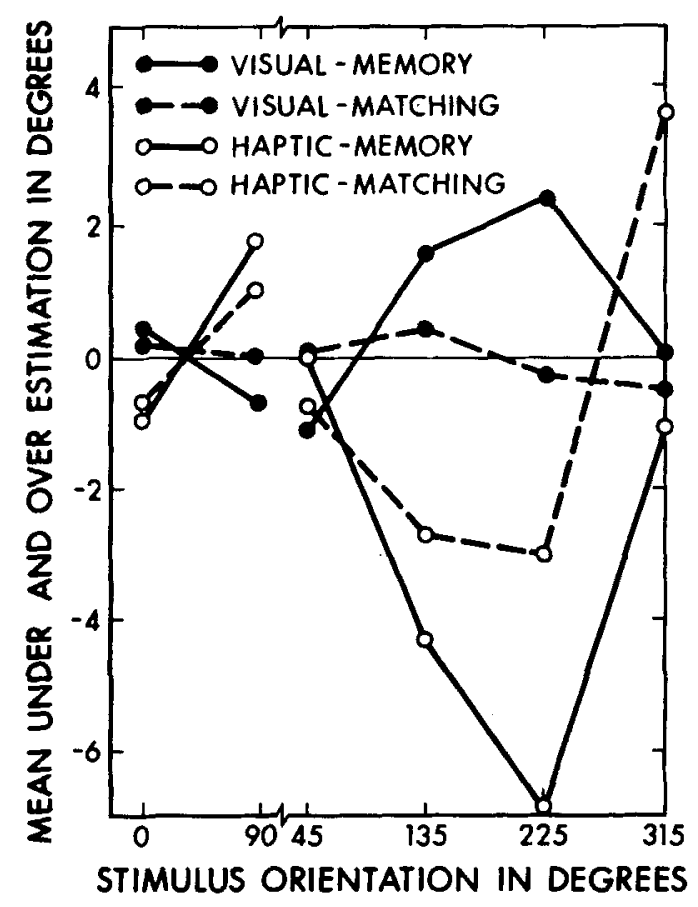

Figure 1. Mean error in produced orientations averaged across both over- and underestimation shown as a function of stimulus orientation for all conditions of modality and response type.

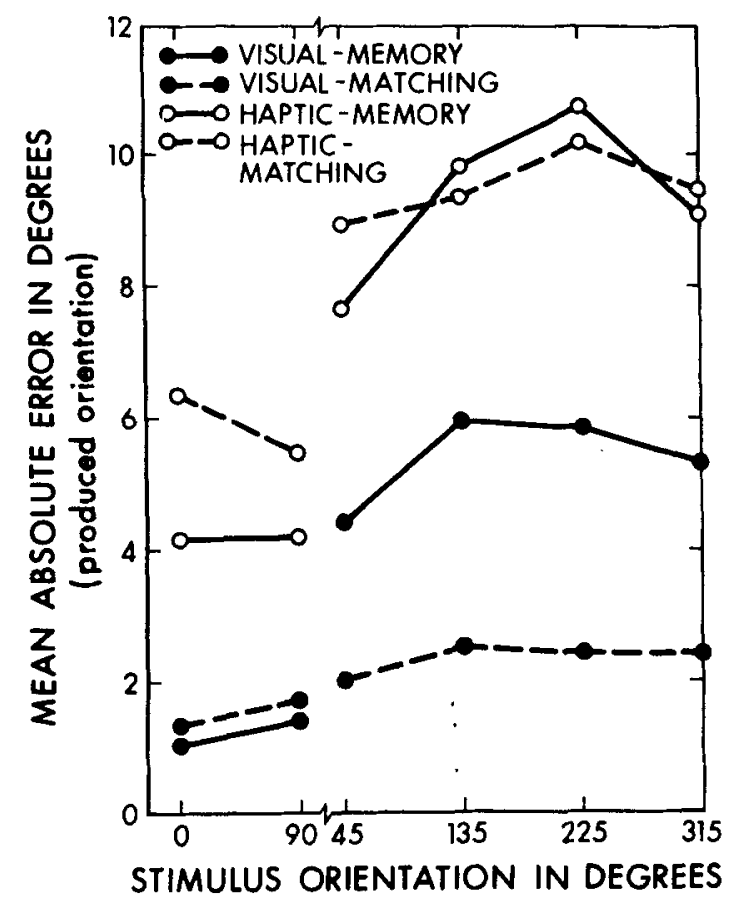

Figure 2. Mean absolute error in produced orientations shown as a function of stimulus orientation for all conditions of modality and response type.

duced more accurately than orientations at quadrant bisections.

As the major interest of the present study was in the accuracy of orientation perception, and since the directionality or sign of errors in produced orientations was very variable and nonsystematic (see Figure 1), it was considered more appropriate to analyze the data in terms of mean absolute errors in produced orientations. Absolute errors reflect better the magnitude of the error, independent of its directionality, since they are not susceptible to cancellation effects when averaging across response errors of opposite sign. An inspection of the two figures shows that when errors in produced orientation are averaged across amounts of over- and underestimation, some 18 data points fall within $2^{\circ}$ of either side of veridical perception (see Figure 1), but when errors are averaged independent of their direction from veridicality, only 5 of the averaged produced orientations are within $2^{\circ}$ of the actual stimulus orientation (see Figure 2).

The functional relationships between stimulus orientation and mean absolute error of produced orientations for all Modality by Response Type conditions are illustrated in Figure 2 and suggest several consistent trends: (1) haptic errors are substantially greater than visual for all treatments; (2) for each Modality by Response Type condition, vertically and horizontally produced orientations are more accurate than productions of all quadrant bisections; and (3) for the visual modality, memory 
errors are greater at all quadrant bisections than simultaneous matching errors.

Data were combined across subjects as preliminary analyses showed neither sex nor order (starting modality) to be significant factors. Also, as the balancing conditions of placing the standard stimulus orientation to the right or left in the matching condition resulted in no systematic differences in produced orientations in either modality, responses were pooled across the two conditions of standard placement.

A 2 (Modality: haptic, visual) by 2 (Response Types: memory, simultaneous matching) by 6 (Orientations: $0^{\circ}, 45^{\circ}, 90^{\circ}, 135^{\circ}, 225^{\circ}, 315^{\circ}$ ) repeated measures ANOVA with Trials being complete replications showed error changes over trials not to be significant, but Orientation, Modality and Response Type all to produce significant main effects $[\mathrm{F}(5,1944)=55.06, \mathrm{p}<.01 ; \mathrm{F}(1,1944)=581.49$, $\mathrm{p}<.01 ; \mathrm{F}(1,1944)=9.78, \mathrm{p}<.01$, respectively]. The two-factor interactions of Orientation by Modality, Orientation by Response Type, and Modality by Response Type were also all statistically significant $[F(5,1944)=4.76, p<.01 ; F(5,1944)=7.82$, $\mathrm{p}<.01 ; \mathrm{F}(1,1944)=39.51, \mathrm{p}<.01$, respectively]. The three-factor interaction, however, was not significant.

The 36 treatment means were compared using Duncan's new multiple range test to test for the significance of the difference between specific treatment conditions. A comparison of visual and haptic modalities for each response type showed modality errors to differ significantly $(p<.01)$ at each orientation, i.e., visual memory and simultaneous matching errors were reliably less than haptic memory and simultaneous matching errors, respectively. Comparisons of errors in produced orientations at each of the stimulus orientations showed that vertical and horizontal errors were significantly less $(p<.01)$ than for all other orientations for each of the four Modality by Response Type conditions. Finally, for the visual modality, errors in produced orientations were greater $(p<.01)$ in the memory than in the simultaneous matching condition for each of the oblique orientations but less at vertical and horizontal orientations. In the haptic modality, the simultaneous matching condition was reliably less accurate than memory $\left(\mathrm{p}^{\circ}<.01\right)$ in producing equivalent orientations at stimulus orientations of $0^{\circ}, 45^{\circ}$, $90^{\circ}$, and $315^{\circ}$, but reliably more accurate $(\mathrm{p}<.01)$ at stimulus orientations of $135^{\circ}$ and $225^{\circ}$.

\section{DISCUSSION}

Results from the present experiment confirm and extend previously reported functional dependencies of spatial discrimination on stimulus orientation (Appelle, 1972). The significant main effects, as illus- trated in Figure 2, demonstrate that: (1) errors in produced orientations, averaged across modalities and response conditions, are significantly different across the stimulus orientations sampled, i.e., there are significant orientational asymmetries; (2) when averaged across stimulus orientations and response conditions, errors in visually produced orientations are reliably different from errors in haptically produced orientations, i.e., there are significant modality differences; (3) the perceptual matching (simultaneous) and memory (successive) response conditions, as defined in this study, result in reliably different magnitudes of error in produced orientation when averaged across stimulus orientations and modalities, i.e., although not comparable in all features except memory, the matching and memory conditions prove to be reliably different in veridically producing orientations; and (4) for each of the four Modality by Response Type conditions, vertical and horizontal orientations were produced reliably more accurately than any of the oblique orientations, i.e., there are significant "oblique effects."

The significance of the main effects need be qualified, however, in light of the significant twofactor interactions. First, the significant Orientation by Modality interaction indicates that the pattern of differences in produced orientations across the stimulus orientations sampled were reliably different for the two modalities. This difference resulted primarily from the significantly greater response error in the haptic modality. When averaged across response conditions, mean errors in haptically produced orientations ranged from $4.81^{\circ}$ to $10.45^{\circ}$ while errors in the visual modality only ranged from $1.55^{\circ}$ to $4.22^{\circ}$. Second, the significant Orientation by Response Type interaction, signifying that errors in produced orientations differed as a function of response type when averaged across modalities, resulted from produced orientation errors varying over more than a threefold range $\left(2.58^{\circ}\right.$ to $\left.8.32^{\circ}\right)$ in the memory condition while not even doubling $\left(3.83^{\circ}\right.$ to $\left.6.27^{\circ}\right)$ in the matching condition. Third, the significant Modality by Response Type interaction implies that the modality main effect is not independent of response type. This interaction resulted from a decrease in average error in produced orientations in the visual modality from $3.99^{\circ}$ to $3.08^{\circ}$ from memory to matching conditions, while haptic errors increased from $7.61^{\circ}$ to $8.25^{\circ}$.

Of greatest significance to the present study was the finding of an "oblique effect" in both modalities, i.e., vertical and horizontal orientations were produced reliably more accurately than any of the other stimulus orientations. Some researchers essentially ascribe an inherent, neurological basis for visual orientational asymmetries and, in particular, the "oblique effect"' (Campbell \& Maffei, 1970; Leehey, Moskowitz-Cook, Brill, \& Held, 1975; Mansfield, 
1974), while others argue in favor of an acquired predisposition to perceive stimuli differently as a function of their physical orientation (see Ross, 1974, and Segall, Campbell, \& Herskovits, 1966). However, to be commensurate with the most recent research demonstrating that specifically restricted early visual experience can functionally modify the nervous system to alter its sensitivity to stimuli in different orientations (Levanthal \& Hirsh, 1975; Stryker \& Sherk, 1975) and that individuals reared in different ecological environments show different orientational anisotropies (Annis \& Frost, 1973), it would seem that both neurological factors and experience are involved in visual spatial perception, including the perception of stimulus orientation.

The present finding of a significant haptic "oblique effect" is of particular interest in that no systematic research has been directed at haptic perception of stimulus orientation, and hence learned or neurological explanations of orientational asymmetries in the haptic modality have not as yet been formulated. However, Mountcastle (1957) did find that the overall pattern of activity of a group of neurons in the cat's somatosensory system signaled joint position and he speculated that the sensibility of limb position was transmitted essentially via the dorsal columnmedial lemniscal system. Although subsequent research has necessitated a reassessment of Mountcastle's results (Wall, 1970), it does appear that the dorsal column "system" is involved in the detection of limb position (Vierck, 1974), in active haptic exploration of the environment (Wall, 1970), and in the sensory-motor integration of concomitant proprioceptive and exteroceptive information resulting from haptic stimulus analysis (Basbaum \& Hand, 1973). Speculatively, it would seem that these studies at least suggest that the detection of the limb position or orientation is based on differential neurological sensitivity to patterns of haptic input varying in tactile-proprioceptive composition and resulting from haptic exploration of stimuli in different spatial orientations. Also, although the effects of haptic experience on haptic stimulus orientation perception have not been explicated, reports of differences in perception of haptic esthetics between blindfolded sighted and congenitally blind subjects (Revesz, 1950; Hintz \& Nelson, 1971) do certainly also suggest at least altered haptic perceptual awareness as a function of differential haptic experience. As with vision, it would thus seem that both experimental and endogenous factors contribute to haptic spatial perception and, in particular, the perception of stimulus orientation.

The magnitude of the "oblique effect" and errors in produced orientations were reliably different for the two modalities. Although haptic and visual physical space are composed of identical stimuli and stimulus arrangements, their phenomenal worlds are certainly discrepant (Cutsforth, 1951). Thus haptic and visual stimulus equivalence need not imply perceptual equivalence (see Brumaghim \& Brown, 1968). Also, the present task was spatial, and studies ranging from spatial acuity to depth perception collectively attest to the eminent superiority of the visual modality in discriminating spatial attributes of stimulation (Gibson, 1966). Revesz (1950) attributes the general inferiority of haptic spatial perception to the inability of the haptic modality to comprehend stimuli simultaneously. He contends that haptic sensory organization and comprehension of the entire stimulus, be it complex shape or the orientation of a rod, involves successive part sampling or proximal contacts over time. Similarly, Cutsforth (1951) and Bartley (1972) maintain that vision and touch represent two entirely different forms of experience with different procedures of stimulus information reception. Bartley (1972, pp. 204-210), for example, contends "that which is seen can be global or extensive . . . but . . . haptic perception . . . is piecemeal and sequential," and Cutsforth (1951, pp. 166-176) states "what is visually simple is tactually complex" because a series of tactile acts is required to achieve what vision can generally achieve instantaneously. Although Gibson (1966) argues for "equivalence of successive sampling and simultaneous grasping" and both stimulus size and complexity do interact with modality to determine the "time course" of perception, given comparable stimulus complexity, vision does appear to have a relative advantage of a more immediate comprehension of the entire stimulus complex. As obtained errors in produced orientations were reliably greater in the haptic modality for both response conditions, the present study adds credence to the contention that haptic assessments of stimulus spatial features is both different from and inferior to visual spatial discrimination (see Geldard, 1970).

Another important result of the present study was the reliable difference between memory and matching response conditions. Interpretation of the significance of this difference, however, must include discussion of the fact that memory vs. matching conditions interacted with both stimulus orientation and modality. Since matched orientations, averaged across modalities, were reliably much more accurate than orientations produced from memory for all oblique stimulus orientations, but slightly less accurate than memory at vertical and horizontal orientations (see Figure 2), it is not surprising that Orientation and Response Type interacted significantly. That both the magnitude of error and difference between response types were less at vertical and horizontal orientations is consistent with both neurological and cultural-learning explanations 
of the "oblique effect," which suggest that humans are equally more "sensitive" to stimuli in vertical and horizontal orientations than oblique orientations, and is also in agreement with Gibson $(1969$, p. 377) that the "frame of reference of vertical and horizontal are characteristic of both the environment and the stimulus array," and hence perception should be more veridical at these orientations.

Previous reports of enhanced orientational asymmetries when using successive stimulus presentation as opposed to a simultaneous stimulus-matching task have been limited to studies of the visual modality in which children were primarily used as subjects and memory for orientation was never directly tested (see Bryant, 1969; Rudel \& Teuber, 1963). The present study also found that (1) errors in produced orientations when adult subjects matched one orientation to that of a standard were less than when they attempted to produce the same orientations only from memory (Response Type main effect), and (2) the difference between simultaneous matching and memory conditions were different for visual and haptic modalities (Modality by Response Type interaction). When averaged across all stimulus orientations, errors in visually produced orientations in the simultaneous matching condition were significantly less than errors in orientations produced from memory, while in the haptic modality memory was slightly superior to the matching condition. It should be pointed out, however, that the "oblique effect" did manifest itself for all four Modality by Response Type conditions (see Figure 2), i.e., vertical and horizontal orientations were produced reliably more accurately than all oblique orientations.

Differences between simultaneous matching and memory conditions are usually discussed in terms of simultaneous vs. successive stimulus presentations, i.e., either stimuli coexist temporally or are presented sequentially. Typically, perceptual discrimination is inferior with the successive condition since the final perceptual report is dependent upon an integration of a series of separate stimulus inputs and thus susceptible to memorial influence (see Bartley, 1972; Cutsforth, 1951). Minnaert (1954) even suggests that some "immediately experienced" spatial illusions are dependent upon a memorial component. The present experiment was primarily interested in comparing simultaneous matching with subjects' memory of specific orientations. The memory condition, as employed in this study, does, however, represent a special condition of successiveness in that subjects were not comparing a "present" stimulus with a specific previous one; rather their responses were based entirely on their memory of specific orientations. An appropriate extension of this research would be to present the stimulus orientation and, after a fixed delay, have subjects produce the same orientation.
The Modality by Response Type interaction seems to reflect the relative advantage vision has in the matching condition where subjects' responses were essentially made in reference to a physically present stimulus. The fact that the mean error in visually matched orientations was reliably less than haptically matched orientations $\left(2.08^{\circ}\right.$ compard to $\left.8.26^{\circ}\right)$ can perhaps be explained in terms of the spatial property of stimulus parallelism (Jastrow, 1893). In adjusting one line to be oriented spatially identical to that of another, as in the matching condition, it is possible that the response is made in terms of linear parallelism rather than matching separate spatial orientations. The perception of stimulus parallelism is certainly relatively more immediately available to vision than to the successive exploration of two stimuli in haptic space for "seldom, if ever, can a tactual perception produce a configuration with the wealth of meaningful relationships that a visual perception produces" (Cutsforth, 1951, p. 170). In the memory condition, where no physical referent was present and subjects had to rely exclusively on their memory of previously perceived orientations, visual errors were again reliably less than haptic $\left(3.99^{\circ}\right.$ compared to $7.61^{\circ}$ ). Since this study used sighted subjects, it seems reasonable to suggest that vision would also have the advantage in the memory condition due to factors of familiarity and past experience. Certainly subjects would have encountered stimuli in different orientations much more frequently visually than haptically. Furthermore, due to the disproportionate preponderance of vertical and horizontal contours over obliques in normal stimulus environments, both modalities should be more accurate in producing these orientations. The data are consistent with both of these expectations and suggest that, as a result of greater visual awareness of stimulus orientation in the everyday experience of sighted subjects, stimulus equivalence need not mean visual and haptic perceptual equivalence.

That visually produced orientations for both response conditions were reliably more accurate than haptic productions is thus consistent with previous research. Also, since absolute spatial discrimination is typically more difficult than relative or relational discrimination in space (Gibson, 1966), the simultaneous matching condition with two coexistent stimuli should yield better orientation discrimination than the memory condition which essentially tested absolute stimulus orientation. However, the data do not support this spatial expectation in that while the mean error in visually produced orientations, averaged across all orientations, decreased from $3.99^{\circ}$ to $2.08^{\circ}$, respectively, haptic mean errors increased from $7.61^{\circ}$ to $8.26^{\circ}$, respectively. Furthermore, the decrease in the difference between modalities in terms of the mean errors of produced orientations in the memory condition $\left(6.18^{\circ}\right)$ compared to the 
matching conditions $\left(3.62^{\circ}\right)$ suggests that the relative advantage of vision in spatial discrimination is dependent upon testing or response conditions.

\section{REFERENCES}

ANNis, R. C., \& Frost, B. Human visual ecology and orientations anisotropies in acuity. Science, 1973, 182, 729-731.

Appelle, S. Perception and discrimination as a function of stimulus orientation: The "oblique effect" in man and animals. Psychological Bulletin, 1972, 78, 266-278.

Bartley, S. H. Principles of perception (2nd ed.). New York: Harper \& Row, 1969.

Bartley, S. H. Perception in everyday life. New York: Harper \& Row, 1972.

Basbaum, A. I., \& Hand, P. J. Projections of cervico-thoracic dorsal roots to the cuneate nucleus of the rat, with observations on cellular "bricks." Joumal of Comparative Neurology, 1973, 148, 347-360.

Braumaghim, S. H., \& Brown, D. R. Perceptual equivalence between visual and tactual pattern perception: An anchoring study. Perception \& Psychophysics, 1968, 4, 175-179.

BRYaNT, P. E. Perception and memory of the orientations of visually presented lines by children. Nature, 1969, 224, 1331-1332.

CAMpbell, F. W., \& MAFFeI, L. Electrophysiological evidence for the orientation and size detectors in the human visual system. Journal of Physiology, 1970, 207, 635-652.

CUTSFORTH, T. D. The blind in school and society. New York: American Foundation for the Blind, 1951.

Geldard, F. A. Vision, audition, and beyond. Contributions to Sensory Physiology, 1970, 4, 1-17.

GrBson, E. J. Principles of perceptual learning and development. New York: Appleton, 1969.

GiBson, J. J. The senses considered as perceptual systems. Boston: Houghton Mifflin, 1966.

Hintz, J. M., \& Nelson, T. M. Haptic aesthetic value of the golden section. British Joumal of Psychology, 1971, 62, 217-223.
JAstrow, J. On the judgment of angles and positions of lines. American Journal of Psychology, 1893, 5, 214-248.

Leehey, S. C., Moskowitz-Cook, A., BRILl, S., \& Held, R. Orientational anisotropy in infant vision. Science, 1975, 190, 900-902.

Leventhat, A. G., \& Hirsch, H. V. B. Cortical effect of early selective exposure to diagonal lines. Science, 1975, 190, 902-904.

MANSFIELD, R. J. W. Neural basis of orientation perception in primate vision. Science, 1974, 186, 1133-1135.

MINNAERT, M. The nature of light and color in the open air. New York: Dover, 1954.

Mountcastle, V. B. Modality and topographic properties of single neurons on cat's somatic sensory cortex. Journal of Neurophysiology, 1957, 20, 508-534.

OVER, R., \& OVER, J. Detection and recognition of mirror-image obliques by young children. Joumal of Comparative and Physiological Psychology, 1967, 64, 467-470.

Revesz, G. Psychology and art of the blind. London: Longmans, Green, 1950.

Ross, H. E. Behaviour and perception in strange environments. London: George Allen and Univin, 1974.

RUDEL, R. G., \& TEUBER, H.-L. Discrimination of direction of line in children. Journal of Comparative and Physiological Psychology, 1963, 56, 892-898.

Segall, M. H., Campbell, D. T., \& Hersxovits, M. J. The influence of culture on visual perception. New York: BobbsMerrill, 1966.

STRYKER, M. P., \& SHERK, H. Modification of cortical orientation selectivity in the cat by restricted visual experience: A reexamination. Science, 1975, 190, 904-906.

VIERCK, C. J., JR. Tactile movement detection and discrimination following dorsal column lesions in monkeys. Experimental Brain Research, 1974, 20, 331-346.

WALL, P. D. The sensory and motor role of impulses travelling in the dorsal columns towards cerebral cortex. Brain, 1970, 93, 505-524.

(Received for publication April 30, 1976; revision received September 24, 1976.) 